Internist 2010 · 51:1085-1085

DOI 10.1007/s00108-010-2726-y

Online publiziert: 20. August 2010

๑) Springer-Verlag 2010

\author{
M. Hellmann - M. Hallek \\ Klinik I für Innere Medizin, Centrum für Integrierte \\ Onkologie Köln-Bonn, Universität zu Köln
}

\title{
Störungen der Thrombozytenfunktion
}

Die Störungen der Thrombozytenfunktion bilden eine wichtige, aber sehr heterogene Gruppe von internistischen Krankheitsbildern. Das klinische Erscheinungsbild reicht von hämorrhagischen Diathesen über mikroangiopathische Störungen wie bei der thrombotisch-thrombozytopenischen Purpura (TTP) bis hin zu schwersten thrombotischen Ereignissen wie beispielsweise im Zuge einer Heparininduzierten Thrombozytopenie (HIT-) II. Interaktionen der Thrombozyten mit Medikamenten stellen ebenso wie (auto-) immunologische Prozesse und angeborene Defekte der Thrombozytenfunktion oft eine Herausforderung in Diagnostik und Therapie dar.

Im pathophysiologischen Verständnis des Gerinnungssystems ist die klassische Gerinnungskaskade als alleiniges Modell inzwischen weitgehend überholt. In der heutigen Vorstellung der Blutgerinnung spielen die Thrombozyten sowohl in der primären als auch in der sekundären Hämostase eine zentrale Rolle, da die Generierung von Thrombin an der thrombozytären Oberfläche stattfindet. Der hohe Stellenwert der Thrombozyten für die Hämostase wird im Beitrag „Pathophysiologie und Biochemie" verdeutlicht. Darüber hinaus zeigen die Autoren die Bedeutung der Thrombozyten für weitere Funktionen, wie beispielsweise bei angiogenetischen und immunmodulatorischen Prozessen, auf.

Störungen der Thrombozytenfunktion laborchemisch korrekt einzugrenzen und zu erfassen, gehört auch heute noch keineswegs zur medizinischen Routine. Die verschiedenen diagnostischen Methoden und ihre klinische Bedeutung für die jeweiligen Krankheitsbilder werden prä- zise und übersichtlich im Beitrag „Stellenwert der Thrombozytenfunktionsdiagnostik“" aufgezeigt. Auch die Bedeutung der Thrombozytenfunktionsdiagnostik zur Therapieüberwachung von kardiovaskulären Patienten unter antithrombozytärer Therapie und das Erkennen von „Nonrespondern“ unter Acetylsalicylsäure- (ASS-) sowie Clopidogrelmedikation sind Themen dieses Beitrags und unterstreichen die fächerübergreifende Bedeutung dieses Wissens.

Im klinisch orientierten Teil dieses Schwerpunkthefts werden zunächst die „klassischen Thrombozytopathien“ und ihre heute gültigen Diagnosekriterien sowie Behandlungsmöglichkeiten beschrieben. Die Ausprägung dieser hereditären Erkrankungen ist dabei ebenso vielfältig wie ihre Ursachen.

\section{O Die Ursachen für „,klassische Thrombozytopathien" sind vielfältig}

Von den klassischen Thrombozytopathien grenzen sich weitere für den Internisten wichtige Krankheitsbilder mit gestörter Thrombozytenfunktion ab. Das Von-Willebrand-Jürgens-Syndrom als häufigste hereditäre Erkrankung mit erhöhter Blutungsneigung stellt ebenso wie die selteneren Erkrankungen HIT-II und TTP mit ihren thrombotischen Komplikationen oft eine große Herausforderung für den internistisch tätigen Arzt dar. Das Erkennen dieser Krankheiten anhand ihrer klinischen Präsentation und eine daraufhin veranlasste zielgerichtete Diagnostik und Therapie sind für die betroffenen Patienten gerade bei diesen Krankheiten oft von vitaler Bedeutung.
Gemäß dem Konzept des Internisten können in einem einzigen Heft nicht alle Aspekte eines derartigen Themas umfassend abgehandelt werden. Daher haben wir uns hier auf die Darstellung von häufig verkannten und therapeutisch anspruchsvollen Krankheitsbildern konzentriert, deren Kenntnisse nicht nur für den hämatologisch oder hämostaseologisch spezialisierten Internisten wichtig sind. In einem Beitrag zur „Arzneimitteltherapie " werden wir zudem in Kürze auch die neuesten Standards zur Therapie der Autoimmunthrombozytopenien zusammenfassen.

Das Schwerpunktheft „Störungen der Thrombozytenfunktion“ soll Ihnen eine aktuelle und praxisorientierte Übersicht $\mathrm{zu}$ diesem Thema geben. Wir wünschen Ihnen eine angenehme und informative Lektüre.

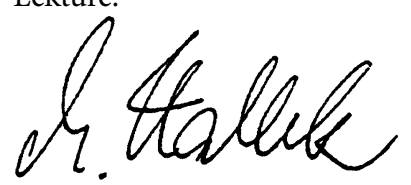

M. Hallek

\section{Korrespondenzadresse}

Prof. Dr. M. Hallek

Klinik I für Innere Medizin, Centrum für Integrierte Onkologie Köln-Bonn, Universität zu Köln Joseph-Stelzmann Str. 9, 50924 Köln

sekretariat-innere1@uk-koeln.de 